\title{
«Estar a la altura de nuestro tiempo»: la televisión educativa, la UNESCO y la modernización de la enseñanza en el franquismo*
}

\author{
Mariano González-Delgado ${ }^{1}$ \\ Universidad de La Laguna \\ mgondel@ull.edu.es
}

RESUMEN: Este trabajo analiza el origen y evolución de la televisión educativa en la España franquista. Se hace especial énfasis en las influencias y la convergencia que el régimen tuvo con organismos internacionales como la UNESCO para la puesta en marcha de esta política curricular. En la primera parte del trabajo se presentan los inicios del modelo curricular descrito. En la segunda, se analiza su posterior evolución. Por último, se intenta dar una explicación sobre el porqué de su desaparición. Este trabajo pretende demostrar que el origen y usos de la TV educativa en España puede ser mejor entendido como un fenómeno que está relacionado con la concepción educativa sobre la que operaba la dictadura. Este aspecto también se vio reforzado por los fenómenos de internacionalización y la convergencia que el franquismo estableció con la teoría de la modernización dentro del contexto de la Guerra Fría.

Palabras Clave: UNESCO; TV educativa; curriculum; teoría de la modernización; Guerra Fría; franquismo.

* Esta investigación ha sido desarrollada bajo el proyecto de investigación «Modernización, desarrollo y democratización. El papel de las potencias europeas occidentales y de las organizaciones internacionales en el cambio político y social en España», Referencia PGC2018097159-B-I00, financiado por el Ministerio de Economía y Competitividad.

Los archivos consultados y sus abreviaturas son los siguientes: Archivo de la Comisión Nacional Española de Cooperación con la UNESCO, Madrid (ACNEC-UNESCO); Archivo Central de la Secretaría de Estado de Educación, Madrid (ACSEE); Archivo General de la Administración, Madrid (AGA).

1 ORCID iD: https://orcid.org/0000-0001-9817-9317.

Copyright: (C) 2020 CSIC. Este es un artículo de acceso abierto distribuido bajo los términos de una licencia de uso y distribución Creative Commons Reconocimiento 4.0 Internacional (CC-BY 4.0) 
«Living up to our time»: Educational television, UNESCO and the modernization of education during Francoism

ABSTRACT: This paper seeks to analyse the origin and evolution of educational television during Franco's rule. Special emphasis is placed on the regime's influence on and engagement with international organizations such as UNESCO regarding the implementation of this curricular policy. The first part of the research deals with the beginnings of the curricular model. The second analyses its subsequent evolution. Finally, we try to provide an explanation for its disappearance. The paper aims to demonstrate that the origin and uses of educational television in Spain can be better understood as a phenomenon that is related to the educational conception on which the dictatorship operated. This aspect was also reinforced by internationalization and Francoist convergence with Modernization Theory in the Cold War context.

KEY WORDS: UNESCO; Educational television; Curriculum; Modernization Theory; Cold War; Francoism.

CÓMO CITAR ESTE ARTÍCULO/CITATION: González-Delgado, Mariano, «"Estar a la altura de nuestro tiempo": la televisión educativa, la UNESCO y la modernización de la enseñanza en el franquismo», Hispania, 80/265 (Madrid, 2020): 597-627. https://doi. org/10.3989/hispania.2020.017.

\section{INTRODUCCIÓN: LA MODERNIZACIÓN EDUCATIVA DURANTE EL FRANQUISMO COMO DEBATE}

Los fenómenos de transferencia educativa como factor que impulsó el proceso de modernización de las políticas educativas de la España contemporánea ha sido un tema tratado de manera amplia por la historiografía educativa ${ }^{2}$. Estas investigaciones han señalado la importancia que tuvo la importación pedagógica en los procesos de cambio y mejora de las estructuras escolares durante el primer tercio del siglo XX en España ${ }^{3}$. Otros periodos de nuestra historia también han producido interesantes estudios en torno a este aspecto. Recientes trabajos han analizado la influencia que ejercieron organismos internacionales como el Banco Mundial (BM), la Organización para la Cooperación y el Desarrollo Económicos (OCDE), o la Organización Mundial de la Salud (OMS) en la transformación del sistema educativo durante la dictadura

\footnotetext{
${ }^{2}$ HERNÁNDEZ DÍAZ, 2016. DEL POZO ANDRÉS, $50 / 5$ (Londres, 2014): 559-614. OTERO-URTAZA, 2013.

3 Véase como ejemplo AGULLÓ DÍAZ y PAYÀ RICO, 7 (Madrid, 2018): 229-334. VIÑAO, extraordinario (Madrid, 2007): 21-44.
} 
franquista ${ }^{4}$. Sin embargo, todavía existen importantes parcelas sin explorar en el estudio de las influencias internacionales en la modernización de la educación durante el franquismo. Aspectos importantes, como la colaboración que la dictadura estableció con la Organización de las Naciones Unidas para la Educación, la Ciencia y la Cultura (UNESCO), apenas han comenzado a estudiarse.

Por otro lado, abrir estas nuevas líneas de indagación histórica permite también pensar en un marco teórico distinto a la hora de abordar el estudio de la educación bajo la dictadura franquista. Las investigaciones previas sobre el franquismo y la educación suelen centrarse en una perspectiva concreta. Lejos de observar los procesos de modernización como una dirección apegada al contexto de su época, la política educativa suele analizarse bajo un espacio de sospecha. De acuerdo con ello, las propuestas educativas lanzadas por el régimen habrían tenido la intención principal de construir el sistema educativo bajo los cauces de los valores tradicionales. El nacionalcatolicismo constituiría el marco central que impregnaría de manera absoluta los planteamientos educativos implementados por la dictadura. Dicho de otro modo, se presentan enfoques en el que los modelos educativos franquistas pivotarían sobre valores religiosos conservadores, sexistas o clasistas ${ }^{5}$. Tales valores constituirían el vector motriz y la causa última que explicarían toda la política educativa llevada a cabo en España durante el franquismo.

Es evidente que los enfoques educativos propios de un Estado autoritario estrechamente hermanado con la Iglesia católica jugaron un papel importante en la transmisión de conocimientos escolares durante el largo periodo franquista. Sin embargo, tal evidencia no excluye la necesidad de pensar el franquismo como un régimen largo que experimentó importantes cambios. En torno a dicha realidad quizá sea necesario hacernos las siguientes preguntas. La política educativa y curricular durante esta etapa, ¿sólo ha presentado valores relativos al nacionalcatolicismo? ¿El nacionalcatolicismo vertebró todo su andamiaje teórico? ¿Constituyó dicha ideología la piedra angular y el marco conceptual fundamental sobre el que se guiaron las políticas educativas entre 1939 y 1975? En respuesta a estas cuestiones conviene señalar que desde los inicios de la década de los cincuenta se pusieron en marcha modelos educativos como la enseñanza programada, la revisión de manuales escolares bajo

${ }^{4}$ Trabajos destacados en este sentido son OSSENBACH y MARTÍNEZ-BOOM, 47/5 (Londres, 2011): 679-700. DELGADO GÓMEZ-ESCALONILLA, 34 (Madrid, 2015): 113146. TERRÓN, COMELLES y PERDIGUERO GIL, 46/2 (Sídney, 2017): 208-223.

${ }^{5}$ Quizá, las investigaciones que de forma más clara se han situado en estos enfoques sean las centradas en el estudio de los manuales escolares durante el franquismo. Véase, como ejemplo, GARCÍA FERNÁNDEZ, 105/1 (Madrid, 2017): 2015-238 y TORNAFOCH-YUSTE y OPISSO ATIENZA, 10/1 (Macerata, 2015): 361-367. 
programas de la UNESCO e incluso proyectos de alfabetización y formación del profesorado con el objetivo de universalizar la enseñanza ${ }^{6}$. Todas estas iniciativas representaban una serie de propuestas curriculares muy cercanas a las políticas educativas nacidas de las teorías de la modernización ${ }^{7}$ y estuvieron muy lejos de presentar un modelo educativo encuadrado bajo las ideas del nacionalcatolicismo.

Hace algunos años, Phillip Jones y David Coleman ${ }^{8}$ señalaron que durante la segunda mitad del siglo XX los sistemas educativos comenzaron a conectarse en el ámbito mundial a través de las redes creadas por los organismos internacionales. En consecuencia, las políticas educativas de distintos países durante esta época comenzaron a mostrar notables similitudes en torno a un concepto de educación muy concreto. Según dicha concepción, las sociedades eran elementos imperfectos que podían generar desigualdades. Para solucionar tales aspectos había que desarrollar elementos institucionales de carácter corrector. Entre ellos, las instituciones educativas podían transformar esa realidad y producir una situación social centrada en algún tipo de cohesión interclasista. De esta forma, al tiempo que la educación podía solventar los problemas de pobreza, también era productora de desarrollo económico. La educación, en definitiva, generaba un capital humano con el que se alcanzarían los objetivos de modernización económica y social ${ }^{9}$.

Por tanto, quizá sea necesario comenzar a analizar la realidad educativa del franquismo desde otra perspectiva. Más que centrarnos en el nacionalcatolicismo como elemento que articuló toda la educación bajo la dictadura, parece pertinente abrir nuevas vías de investigación que presten mayor atención a las conexiones e interacciones educativas a nivel internacional y transnacional, así como a la propia concepción que el régimen tenía de la educación y la idea de sociedad. Ello nos permitíría entender que la ideología del nacionalcatolicismo estuvo presente, pero que no entraba en contradicción con otro tipo de propuestas educativas conectadas con actores, prácticas y discursos que transcendían el marco nacional. El error quizá ha partido del supuesto de que la ideología del nacionalcatolicismo fue totalizadora en la educación franquista, el franquismo era un régimen completamente aislado internacionalmente y que ello no permitió introducir otro tipo de proyectos curriculares.

El propósito de este artículo es analizar el origen y evolución de la televisión educativa en el franquismo. El inicio de este modelo curricular ofrece un buen ejemplo del interés y la aplicación, por parte del régimen, de las políticas curriculares canalizadas por los organismos internacionales para producir procesos

\footnotetext{
${ }^{6}$ GONZÁLEZ-DELGADO y GROVES, 4/2 (Salamanca, 2017): 73-100.

7 MARTÍN GARCÍA, 34 (Madrid, 2015): 27-52.

8 JONES y COLEMAN, 2006.

9 TRÖLHER, 5/2 (Londres, 2013): 1-19.
} 
de modernización educativa durante este periodo. De forma más específica, se señala como la UNESCO, en relación con la OCDE, generó una serie de ideas educativas que la dictadura consideró como deseables y necesarias para que España adquiriese (al igual que los países occidentales) nuevas perspectivas de tipo funcionalista y técnico para el ámbito educativo. Este trabajo, por tanto, pretende demostrar cómo entre 1952 y 1975 los modelos curriculares pueden ser interpretados como una posible concreción de los fenómenos de internacionalización educativa. En este sentido, esta investigación intenta señalar que la concepción sobre el sistema educativo manejada por el franquismo fue similar a las premisas que habían sido definidas por los citados organismos internacionales. Incluso, se podría afirmar la existencia de notables confluencias con las ideas transmitidas por los mismos. La TV educativa, por tanto, es analizada como una política curricular encuadrada bajo el espacio más amplio de las teorías de la modernización y del contexto de la Guerra Fría. Este sería el marco de análisis que nos puede ayudar a entender el origen, dirección y evolución de la TV como herramienta educativa en esta época.

\section{LOS INICIOS DE LA TV EDUCATIVA EN ESPAÑA: UN FENÓMENO MEDIADO POR LAS INICIATIVAS DE LA UNESCO}

En 1952, el Ministerio de Educación Nacional (MEN), publicó un artículo en la sección final de la Revista de Educación denominada «Crónicas» ${ }^{10}$. Parecía ser un artículo menor. Su autor trataba de resumir y hacer suyos los argumentos esgrimidos por la UNESCO en un congreso celebrado en Milán ese mismo año sobre «Los Métodos Audiovisuales en la Educación Fundamental». El autor señalaba que «el analfabetismo no es sólo un problema exclusivamente cultural». El mismo remitía o se relacionaba con problemas relativos a «factores de índole económica». A su vez, todos los fenómenos relacionados con la «desnutrición», la «incultura» o la «justicia social» podían achacarse también a la falta de educación y al analfabetismo. En este sentido, el autor aceptaba que el analfabetismo venía a ser sinónimo de pobreza. Por tanto, era necesario «precisar con mayor urgencia» la extensión de los programas de educación fundamental en España para buscar solución «al problema del analfabetismo y a la mejora inmediata del nivel de vida y de producción de los más amplios y distintos sectores de la población rural». Sin embargo, había que buscar una fórmula lo más eficiente posible para resolver la cuestión. Se argumentó que dicha fórmula estaba en los «métodos audiovisuales», ya que los mismos

\footnotetext{
${ }^{10}$ DE REYNA, 1/2 (Madrid, 1952): 164-167.
} 
ofrecían una «eficacia indiscutible» ${ }^{11}$ en los procesos de alfabetización y aprendizaje.

Al año siguiente, esta misma revista publicó otro artículo sobre medios audiovisuales y enseñanza ${ }^{12}$. Esta vez, hacía referencia directa a la TV educativa. Se trataba de un trabajo que analizaba la primera universidad que ponía en marcha un modelo de TV para sus estudiantes: la Georgetown University de Washington. Este ensayo, intentaba hacer ver las grandes «posibilidades educativas de la televisión». Su autor señalaba, una y otra vez, que el modelo que se presentaba en Georgetown se mostraba como una amplia experiencia de progreso y modernidad que abría las puertas al «mundo del futuro». No obstante, también se presentaban algunas «objeciones de carácter pedagógico». Es importante indicar que el autor no parecía estar de acuerdo en que la TV educativa se formulase como «sustituto del profesor, lo cual parece un error». A pesar de ello, el artículo acababa concluyendo que la TV educativa parecía una fórmula llena de «esperanza» y que había que tener presente los «efectos educativos que preconizaba $\rangle^{13}$. De esta forma, el debate acerca de este modelo curricular comenzaba a abrirse camino en España como propuesta pedagógica.

Lo importante a destacar de estos artículos es que se centraron en dos ideas esenciales: educación y desarrollo y modernización. Así, las primeras referencias que aparecían sobre TV educativa en España estaban directamente encuadradas en los principios de «alfabetización funcional» hacia los que había girado la UNESCO, fruto de una lucha abierta con el Banco Mundial (BM). A finales de los años cuarenta el BM propuso a la UNESCO que abandonase las campañas de alfabetización básica centradas en la concientización. Según el $\mathrm{BM}$, la UNESCO tendría que reorientar este programa hacia una alfabetización de masas que se centrase en el crecimiento económico y la modernización de los países empobrecidos ${ }^{14}$. De esta manera, cuando el Programme of Fundamental Education ${ }^{15}$ llegó a España, la dirección parecía estar clara. El proceso de modernización se había hecho un hueco y, a principios de los años sesenta, ya sería la principal orientación que la UNESCO pondría en práctica.

Por otro lado, y en lo que al contexto de la dictadura se refiere, el inicio de la década de los cincuenta del siglo XX supuso el comienzo de la apertura internacional. En 1953, la España franquista firmaba una serie de acuerdos bilaterales con EE. UU. Es cierto que dichos acuerdos para establecer bases militares en España estuvieron sujetos a un contexto incómodo y contradictorio. EE. UU. se presentaba al mundo como el promotor de la sociedad abierta y

\footnotetext{
${ }^{11}$ DE REYNA, 1/2 (Madrid, 1952): 164 y 165.

12 DE SOBRINO, 4/9 (Madrid, 1953): 47-49.

13 DE SOBRINO, 4/9 (Madrid, 1953): 49.

14 DORN y GHODSEE, 36/2 (Oxford, 2012): 375.

15 BOEL, 2016.
} 
la democracia liberal en su carrera por evitar el crecimiento geopolítico de la Unión Soviética en el transcurso de la Guerra Fría. Sin embargo, el espacio geopolítico abierto por la misma permitió justificar tales acuerdos con el país ibérico desde una óptica de tipo militar y económica. El resultado sería la instalación de bases militares en España, pero también una serie de ayudas económicas que intentaban mejorar el desarrollo económico del país europeo ${ }^{16}$.

En torno a dichas ayudas, se iniciaron una serie de programas de intercambio y formación de capital humano que tenían por objetivo mejorar algunos elementos de las estructuras administrativas de España. Cuadros dirigentes del aparato estatal, así como militares, técnicos, especialistas educativos, responsables de medios de comunicación o estudiantes comenzaron a recibir formación de diferente tipo en el país norteamericano ${ }^{17}$. El acercamiento institucional permitió que la teoría de la modernización se familiarizase con la cúpula franquista $^{18}$ y abriese la carrera de España hacia la aproximación a las medidas de liberalización económica ${ }^{19}$. Este aspecto nos permite entender mejor que el proceso de modernización no sólo se estaba iniciando en el espacio militar, cultural o económico. El ámbito educativo también fue objeto de notables transformaciones dentro de dicho contexto.

De esta forma, después de la publicación de los primeros artículos relativos a la TV educativa, en los años sucesivos comenzarían a aparecer trabajos en esta dirección de forma más numerosa. 1958 supuso un año especial en este sentido. Por un lado, comenzaron a aparecer trabajos mucho más acabados sobre lo que significaba la TV dentro del espacio escolar en sentido estricto. La Revista de Educación publicaba tres artículos de Jacques Bousquet quien, a la postre, sería designado Advanced Training Programme (ATP) UNESCO en España con la creación del Centro Nacional de Investigaciones para el Desarrollo de la Educación (CENIDE). Más adelante volveremos sobre ello. Los dos primeros artículos se presentaban de manera separada, pero en realidad formaban parte de una publicación conjunta. En ellos, Bousquet reflexionaba sobre el sentido último de la TV en las aulas. Se preguntaba, si la TV podía «reemplazar al maestro tradicional». Dicha reflexión no se expresaba con el objetivo de «sustituir al maestro». Simplemente, se quería dejar constancia de que el uso de la TV en las aulas aspiraba a «mejorar las condiciones laborales de los maestros y las técnicas didácticas»; a que «todo el mundo pueda adquirir fácilmente una instrucción de tipo secundario [enseñanza secundaria] $\rangle^{20}$; y a que se

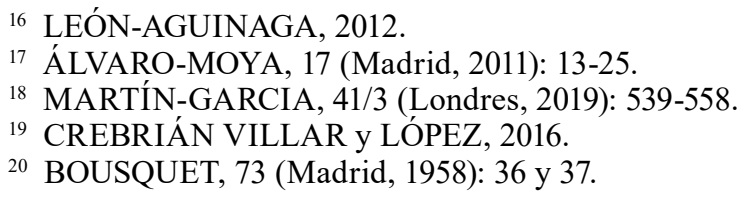


pudiera «eliminar la diferencia entre el público urbano y el rural $»^{21}$. Además, en el tercer artículo ${ }^{22}$, este mismo autor insistiría sobre la cuestión de la TV educativa. Dicho elemento técnico podía ayudar a abaratar el «precio de la educación» a los sistemas educativos en vías de desarrollo y a alcanzar la meta de la escolarización universal para conseguir una mayor modernización económica.

Al mismo tiempo, la revista Bordón, dependiente de la Sociedad Española de Pedagogía (SEP), publicaba un monográfico dedicado a «Televisión y Radio Escolar». En él, se podían encontrar artículos en los que se utilizaban los argumentos descritos anteriormente ${ }^{23}$. La mayoría de los trabajos que aparecieron en dicho número especial presentaron diferentes modelos de televisión y radio escolar que se llevaban a la práctica en países como Francia, Inglaterra o Colombia $^{24}$. Todos ellos eran casos relacionados con experiencias de la UNESCO. Sin embargo, uno de los artículos de este monográfico desarrollaba una serie de críticas a la TV educativa. Su autor se preguntaba si esta podría «suplantar la función del maestro». A pesar de ello, más adelante se expresaba que en realidad la TV ejercía una «función de complemento» y que «sin el concurso del maestro la emisión ha de ser forzosamente ineficaz» $»^{25}$. Sin embargo, este tipo de críticas no representaban limitaciones nacidas del contexto español: en realidad, solo reproducían las advertencias que la revista $\mathrm{El} \mathrm{Correo} \mathrm{de}$ la UNESCO había publicado unos años antes en un número especial sobre «La Televisión: oportunidad y problemas educativos $\rangle^{26}$. Sea como fuere, la TV educativa se presentaba como una fórmula válida para abordar aquellas cuestiones estructurales que preocupaban a la UNESCO: alfabetización y universalización de la enseñanza como fórmula para alcanzar el desarrollo económico.

Por otro lado, también comenzaron a aparecer otro tipo de trabajos que complejizaba aún más la cuestión. La TV educativa, no sólo se relacionaba con una fórmula para superar el analfabetismo. Los principios educativos que podían ser trabajados con el alumnado eran múltiples. La TV podía funcionar como método curricular que ayudase a poner en práctica en España el International Understanding Programme de la UNESCO para fomentar el «conocimiento y la comprensión mutua de las naciones $»^{27}$. Además, también se asoció con intentos claros por modernizar el espacio del aula a través de la «reforma»

${ }^{21}$ BOUSQUET, 74 (Madrid, 1958): 64.

${ }^{22}$ BOUSQUET, 88 (Madrid, 1958): 25-29.

${ }^{23}$ FEMENÍAS LOYOLA, 78-79 (Madrid, 1958): 415-424.

${ }^{24}$ DIEUZEIDE, 78-79 (Madrid, 1958): 405-414. TAYLOR, 78-79 (Madrid, 1958): $395-$ 404. WARLETA FERÁNDEZ, 78-79 (Madrid, 1958): 459-468.

${ }^{25}$ COSTA RIBA, 78-79 (Madrid, 1958): 377.

${ }^{26}$ UNESCO, 6/3 (París, 1953).

27 BORREGUERO SIERRA, 147 (Madrid, 1962): 31. 
de los viejos libros de texto como las «Enciclopedias» ${ }^{28}$, y la «revisión de los métodos didácticos tradicionales $\rangle^{29}$. Incluso, durante estos primeros debates se pudieron escuchar algunas importantes voces del campo educativo español que vieron en la TV un espacio para, junto con la Sociología de la Educación o la Educación Comparada, reivindicar la relación entre la escuela y el «desarrollo económico» ${ }^{30} \mathrm{y}$ hacer «científica la reflexión educativa» o modernizar la investigación pedagógica. En definitiva, hacerla pivotar de «la etapa artesanal a la etapa técnica $)^{31}$.

De esta forma, en este segundo grupo de artículos, las discusiones acerca de la TV educativa aparecían de una manera más cercana a la práctica. En realidad, los mismos se encuadraban ya dentro de las iniciativas para desarrollar el Programme of Fundamental Education de la UNESCO en España. En 1953, el país ibérico firmó los acuerdos para ser miembro de pleno derecho de la $\mathrm{UNESCO}^{32}$. Ello propició dos aspectos. El primero, hacía referencia a la creación de la Comisión Nacional Española de Cooperación con la UNESCO y, con ella, la gestión de una serie de seminarios promovidos por la UNESCO en España para desplegar el Proyecto Principal número uno de Educación para América Latina. Dichos seminarios fueron de naturaleza distinta. Se pudieron observar temas tan variopintos como Curso de Formación de Técnicos en Estadistica Educativa, Curso sobre Escuelas Unitarias Completas, Curso de Construcciones Escolares o un Curso-Coloquio sobre el Planeamiento Integral de la Educación ${ }^{33}$. Lo importante a destacar aquí es que se pudieron observar algunas publicaciones relativas al uso de medios audiovisuales y la modernización educativa ${ }^{34}$. La segunda, que dos instituciones educativas como la Junta Nacional contra el Analfabetismo y la Comisión para la Extensión Cultural ajustaron cada vez más sus objetivos en dirigir el espacio educativo hacia los procesos de alfabetización funcional. De esta manera, la Junta Nacional contra el Analfabetismo creó un programa de alfabetización para militares en el que se usaron métodos audiovisuales para intentar hacer más eficiente el aprendizaje de la lectura y la escritura ${ }^{35}$. Asimismo, la Comisión de Extensión Cultural traducía y adaptaba al español una serie de Cuadernos de Educación Fundamental publicados por la UNESCO, con el fin de «poner todos los medios a su

${ }^{28}$ GONZALVO MAINAR, 92-93 (Madrid, 1960): 248.

29 GARCÍA JIMÉNEZ, 21/82-83 (Madrid, 1963): 55.

${ }^{30}$ GARCÍA HOZ, 107-108 (Madrid, 1962): 127.

31 MAÍLLO, 149 (Madrid, 1963): 118.

32 Sin embargo, desde 1951, España comenzó a acudir como observadora invitada de las diferentes Conferencias organizadas por la UNESCO. OLIVEROS, 1978.

${ }_{33}$ MEN, 1962: 5-18.

34 NAVARRO HIGUERAS, 1960.

${ }^{35}$ MAÍLLO, 54 (Madrid, 1955): 371-374.

Hispania, 2020, vol. LXXX, n. ${ }^{\circ}$ 265, mayo-agosto, págs. 597-627, ISSN: 0018-2141, e-ISSN: 1988-8368 https://doi.org/10.3989/hispania.2020.017 
alcance, audiovisuales y publicaciones», para «alfabetizar las zonas rurales» ${ }^{36}$. A ello, habría que sumar las primeras iniciativas de la Televisión Pública en España. Televisión Española (TVE) creó, entre 1958 y 1966, una serie de programas que tenían por objetivo intentar ayudar a erradicar el analfabetismo. A pesar de no estar cohesionadas y gestionadas directamente por el sistema escolar y el MEN, programas como Aula TV, Lecciones de Inglés, Lecciones de Francés, Imágenes para saber, Televisión Escolar, Aula TV o Universidad TV ${ }^{37}$ se originaron con relación a las iniciativas que los organismos internacionales habían recomendado a España en torno a la cuestión del analfabetismo.

En este mismo sentido, el debate acerca de la necesidad de aumentar los procesos de alfabetización con el uso de la televisión también alcanzó al ámbito extraescolar. En 1964 el Ministerio de Información y Turismo creó la Red Nacional de Teleclubs ${ }^{38}$. El proyecto pretendía llevar la TV a las zonas rurales de España. Los aparatos televisivos se situarían en espacios públicos de los pueblos para que sus habitantes acudiesen a ver los programas que se emitían por televisión. Se trataba de un proyecto que pretendía acerca la cultura a los ámbitos rurales con el fin de romper la distancia respecto al espacio urbano. Lo importante a señalar aquí, es que los Teleclubs no fueron un invento español. En realidad, era una importación de un modelo educativo que la UNESCO había supervisado en diferentes países como Francia o Japón ${ }^{39}$ para intentar acabar con el analfabetismo en las zonas rurales.

No obstante, no sólo la cuestión sobre el analfabetismo y la mejora de las técnicas pedagógicas originó y encuadró la introducción de la TV educativa en España. La idea en torno a la importancia que la educación debía tener para el desarrollo económico también delimitó su inicio y direcciones. En diciembre de 1961, la OCDE, en colaboración con el propio régimen, constituyó un grupo de especialistas con el fin de analizar «las necesidades españolas a largo plazo en materia de enseñanza» ${ }^{40}$. En las conclusiones del informe, la OCDE señalaba que España tenía que emprender un «vasto programa de desarrollo de la enseñanza» con el fin de «duplicar su producto nacional bruto entre 1961 y 1975». Para lograrlo, la OCDE era clara en sus propuestas. España debía «ampliar el acceso a la enseñanza de las clases con ingresos inferiores»; «reducir la desigualdad existente entre unas regiones y otras en el acceso a la cultura»; «ampliar el acceso a la enseñanza de las mujeres»; y «reducir el descrédito escolar resultante del abandono prematuro». El objetivo, en definitiva, era

36 GARCÍA, 196 (Madrid, 1960): 207.

37 ANTONA, 20 (Madrid, 2014): 209-226. OSSENBACH y GROVES, 11 (París, 2013): 2-17.

${ }^{38}$ MARTÍN ANTÓN, 29 (Madrid, 2017): 353-390.

39 DUMAZADIER, 1956. CASSIRER, 6/12 (París, 1953): 17-18.

40 OECD, 175 (Madrid, 1965): 117. 
«modificar profundamente la estructura cuantitativa y cualitativa de la población nacional activa $»^{41}$ a través del sistema educativo y conseguir con ello una ampliación del capital humano que produjese, a su vez, un mayor desarrollo económico.

Sin embargo, las propuestas de la OCDE no terminaron en este punto. En una ampliación de ese mismo informe, en conjunto con el BM, ambas instituciones extendían el espacio de recomendaciones para España. Se trataba de la puesta en marcha de un proyecto para desarrollar los países del sur de Europa. Nos referimos al Proyecto Regional Mediterráneo ${ }^{42}$. En dicho informe, la OCDE y el BM señalaron que «proveer acomodación para todos los niños» en el sistema escolar era un plan totalmente necesario. No obstante, «la conexión entre la educación y el desarrollo económico es tan importante que el informe no sería completo sin alguna referencia a este asunto». Así, para estos organismos internacionales, la inversión para el «aumento cuantitativo» de la «educación primaria», «la educación técnica», «el nivel universitario»o «la formación profesional para adultos», era un asunto importante. Pero también «lo es el aumento en calidad». Era necesario que el sistema educativo español modificase la «desastrosa carencia de material de enseñanza» ${ }^{43}$. Es decir, utilizar las nuevas técnicas didácticas, como la TV educativa, con el fin de conseguir ese aumento de escolarización, al tiempo que se mejoraba la «calidad» de la enseñanza. La teoría de la modernización, por tanto, se comenzaba a manifestar como uno de los principios básicos que orquestaría el modelo curricular durante el franquismo. Un aspecto que no nos debe extrañar en absoluto. Unos años antes, la Revista de Estudios Políticos había publicado una serie de artículo de uno de los máximos exponentes de la teoría de la modernización, Seymour M. Lipset $^{44}$.

De esta forma, comenzaron a surgir propuestas enmarcadas dentro de tales objetivos. En 1963 se estableció el primer modelo de TV educativa adscrito directamente al MEN. Se trató del Centro Nacional de Enseñanza Media por Radio y Televisión, más conocido como Bachillerato RTV. Dicho centro venía a ser una evolución del Bachillerato Radiofónico que un año antes había creado el propio MEN para extender la enseñanza media ${ }^{45}$. Entre sus objetivos principales estaba llevar «hasta sus últimas consecuencias los principios de extensión de la enseñanza y de igualdad de oportunidades para todos los españoles» ${ }^{46}$.

${ }^{41}$ OECD, 175 (Madrid, 1965): 117.

${ }^{42}$ OECD, 147 (Madrid, 1962): 25-30. Más información en DELGADO GÓMEZ-ESCALONILLA, 34 (Madrid, 2015): 113-146 y CORRALES MORALES, 2020.

${ }^{43}$ OECD, 147 (Madrid, 1962): 26 y 27.

${ }^{44}$ LIPSET, 100 (Madrid, 1958): 49-94; LIPSET, 139 (Madrid, 1965): 5-40.

45 Orden de 9 de noviembre de 1962 (BOE de 28 de noviembre de 1962).

46 Decreto 1181/1963 de 16 de mayo, para el establecimiento del Centro Nacional de Enseñanzas Medias por Radio y Televisión (BOE de 1 de junio de 1963). 
Para conseguirlo, el ministro de Educación Manuel Lora Tamayo, señaló que «las modernas técnicas audiovisuales» eran las herramientas adecuadas. Y que, incluso, las mismas estaban «llamadas a ejercer un papel importante en la elevación del nivel cultural de nuestro pueblo, imprescindible para acometer las empresas de desarrollo que ya urgen $\rangle^{47}$.

Lo importante a destacar de esta iniciativa es que Bachillerato RTV estaba integrado directamente en las recomendaciones que los organismos internacionales habían planteado a España durante estos años. El principio de igualdad de oportunidades no era un aspecto gratuito o un mero discurso hecho por el régimen de cara a la galería. En realidad, tal principio venía a ser una condición previa fundamental para conseguir el objetivo del desarrollo económico. Un aspecto que la TV educativa podía ayudar a conseguir.

Por estos motivos, no nos debe extrañar que España se adhiriera al modelo curricular aquí descrito. En realidad, la UNESCO ya había insistido de manera amplia en la necesidad de usar la TV como forma de paliar el analfabetismo y crear procesos de desarrollo económico en otros países. Algunos años antes de la creación del Bachillerato RTV en España, la UNESCO ya publicaba una serie titulada Reports and Papers on Mass Comunication. En ella, se incluían trabajos acerca del uso de la TV educativa en diferentes zonas rurales de países europeos, en países asiáticos o, incluso, cómo los medios audiovisuales podían fomentar los conocimientos de $\operatorname{arte}^{48}$. No obstante, los esfuerzos de la UNESCO no se centraron exclusivamente en estas regiones. La TV educativa podía ser un método adecuado para solucionar los problemas de infraestructura que podían tener algunas de las zonas geográficas más pobres del mundo. En una reunión sobre «Educational Broadcasting in Tropical Africa», que tuvo lugar en 1961 en Moshi, Tanganica, los países miembros de la UNESCO llegaron a un acuerdo. Debido a la «falta general de maestros» ${ }^{49}$ que tenía el continente africano, diferentes países como Marruecos, Kenia o Nigeria, debían intentar establecer servicios regulares de TV educativa. Además, desde principios de los años sesenta la UNESCO ya había comenzado a desarrollar también otros proyectos en países árabes ${ }^{50}$. La TV educativa se erigía, bajo el marco de los organismos internacionales, como un modelo curricular válido y necesario para intentar conseguir los propósitos de modernización económica en distintos países a nivel mundial.

\footnotetext{
47 «Editorial», 1 (Madrid, 1963): 1.

48 UNESCO, 1960; 1961.

49 Meeting on Educational Broadcasting in Tropical Africa, Moshi, Tanganika (1961) EDBC/ AFR/5; WS/0861.95, http:/www.unesco.org/ulis/cgi-bin/ulis.pl?catno=144689\&set=005BC9BB9F_1_417\&gp=1\&lin=1\&ll=1: 4.

${ }_{50} \bar{E}$ Eucational television in Pakistan, (mission), 4-23 October 1960; report WS/0961.113, http://www.unesco.org/ulis/cgi-bin/ulis.pl?catno=156962\&set $=005$ BC9BC7E_3_448\&$\mathrm{gp}=1 \&$ lin $=1 \& 11=1$
}

Hispania, 2020, vol. LXXX, n. ${ }^{\circ}$ 265, mayo-agosto, págs. 597-627, ISSN: 0018-2141, e-ISSN: 1988-8368 https://doi.org/10.3989/hispania.2020.017 
En este sentido, España no aparecía como un islote educativo. Era uno más de los países que manejaba la misma concepción que utilizaba la UNESCO, la OCDE y el BM acerca del uso del sistema educativo como ámbito de modernización. La TV educativa, por tanto, no se desplegaba como un modelo curricular en abstracto. En realidad, su origen tenía que ver con los intentos por conseguir ampliar la enseñanza en las distintas clases sociales, mejorar los procesos de enseñanza y aprendizaje y, de una manera más amplia, conseguir que España se introdujese en la senda del desarrollo económico propuesto por los organismos internacionales. Se trataba de dar una solución a problemas sociales (pobreza, desempleo, igualdad y educación) a través de elementos de carácter técnico. En el fondo, la TV educativa en España se enmarcaba en los planes de los países occidentales por intentar mejorar las condiciones de vida de los países en vías de desarrollo. Un tipo de política curricular enmarcada, de manera más amplia, dentro de los debates de la Guerra Fría sobre la modernización educativa.

\section{LA AMPLIACIÓN DE LA TV EDUCATIVA COMO MODELO CURRICULAR}

En torno a estos debates sobre educación y desarrollo, pronto comenzaron a aparecer los primeros trabajos españoles sobre TV educativa centrados en esta cuestión. En 1963, el que a la postre fuese director de la Radio-Televisión Educativa, Jesús García Jiménez, publicaba un artículo en un monográfico de la revista Bordón dedicado a «Educación y Desarrollo Económico». El título del artículo, «Medios audiovisuales y cultura del desarrollo», estaba lleno de referencia a autores, libros e informes publicados por la UNESCO sobre este tema particular. Todo ello, venía a refrendar la importancia que comenzó a tener en España la idea de que «los medios de difusión» eran centrales para «el incremento de la productividad nacional $\rangle^{51}$.

Sin embargo, el intento por socializar el uso de los medios audiovisuales en el aula no se quedó exclusivamente en el ámbito del debate académico. El Centro de Documentación y Orientación Didáctica de Enseñanza Primaria (CEDODEP), como organismo encargado de la formación y perfeccionamiento del magisterio, comenzó a desarrollar propuestas para instaurar el uso de la TV en los centros educativos. Durante los primeros años sesenta, la revista Vida Escolar publicó una serie de experiencias para que los métodos audiovisuales comenzasen a usarse de manera más sistemática en las aulas de Enseñanza Primaria. Trabajos sobre la reflexión de un «uso eficiente» por parte del magisterio, la aplicación de «la grabación magnética» o «el magnetófono», empezaron a ser habituales en sus

\footnotetext{
${ }^{51}$ GARCÍA JIMÉNEZ, 21/82-83 (Madrid, 1963): 381.
} 
páginas $^{52}$. Incluso, el CEDODEP crearía un concurso entre el magisterio con el fin de motivar el uso de los medios audiovisuales en las aulas ${ }^{53}$.

No nos deben extrañar en absoluto las rápidas iniciativas por intentar socializar este modelo curricular entre el profesorado español. La publicación de estos primeros ejemplos prácticos se producía en paralelo al compromiso que el MEN había adquirido ante la UNESCO para utilizar este tipo de medidas. En una intervención del día 26 de octubre de 1964 ante la XIII Conferencia Internacional de la UNESCO, el ministro de Educación Nacional, Lora Tamayo, venía a reconocer que «el Gobierno español continuará participando activamente en las diversas actividades que constituyen el programa de la UNESCO», entre las que destacaba «el problema de la formación del profesorado en todos sus grados». Dicho compromiso no hacía más que refrendar el que se había producido poco antes con la celebración en España del $I^{e r}$ Seminario Iberoamericano de Alfabetización, en coordinación con la UNESCO. En el mismo, se creó una mesa específica para trabajar sobre «el empleo de los medios audiovisuales e informativos en general» en las aulas ${ }^{54}$.

Dentro de este clima educativo, pronto comenzaron a aparecer las primeras experiencias sobre el uso de la TV en las aulas de enseñanza primaria. Todas ellas, se presentaron en el III Congreso Nacional de Pedagogía, celebrado en Salamanca en el año 1964. El propio título del congreso, El sistema escolar español en función de las necesidades económicas y sociales, daba la medida de dónde se insertaban estos primeros trabajos acerca de la TV educativa. Raquel Payà Ibars presentaba una experiencia sobre el uso de la TV en una serie de centros escolares de Madrid. Después de utilizar la TV para enseñar contenidos de matemáticas, ciencias sociales o geografía a alumnado entre $9 \mathrm{y}$ 11 años, la autora concluía que «la televisión es una de las técnicas audiovisuales de decisiva importancia en la formación y promoción cultural, profesional y social de los hombres $\rangle^{55}$. La importancia y el atractivo que la imagen podía tener para adquirir los conocimientos y habilidades necesarias para el mundo laboral también eran refrendados por otras experiencias llevadas a cabo en el mismo periodo ${ }^{56}$. En realidad, estas iniciativas fueron fruto de la creación por parte del MEN y la Dirección General de Información de un Grupo de Estudio sobre las Técnicas Audiovisuales (GESTA). El objetivo, era investigar y promocionar la TV en el aula para intentar que dicha técnica vertebrase los

52 NAVARRO HIGUERAS, 62 (Madrid, 1964): 26-28; 65 (Madrid, 1965): 19-21. MALLAS CASAS, 37 (Madrid, 1962): 32-34.

53 «Primer concurso...», 68 (Madrid, 1965): 33.

${ }^{54}$ LORA TAMAYO, 166 (Madrid, 1964): 55 y 59.

55 PAYÀ IBARS, 23/91-92 (Madrid, 1965): 661.

56 SUÁREZ RODRÍGUEZ, 23/91-92 (Madrid, 1965): 760-761. MARTÍN ALONSO, 23/91-92 (Madrid, 1965): 614-615.

Hispania, 2020, vol. LXXX, n. ${ }^{\circ}$ 265, mayo-agosto, págs. 597-627, ISSN: 0018-2141, e-ISSN: 1988-8368 https://doi.org/10.3989/hispania.2020.017 
procesos de aprendizaje, dado que «la enseñanza por medio de la televisión tiene mayor valor formativo que la enseñanza directa $\rangle^{57}$.

Los intentos por promover la TV en las aulas no se detuvieron en este punto. La investigación educativa, como fórmula para mejorar la práctica docente, empezó a ser un espacio que también necesitaba reforzarse para fomentar su uso. En el discurso de clausura del citado III Congreso Nacional de Pedagogía, el presidente de la SEP y del Instituto San José de Calasanz de Pedagogía, Víctor García Hoz, así lo ponía de manifiesto. En el mundo actual, «la progresiva tecnificación de la vida social implica la mayor intervención de factores científicos» con el propósito de adecuar, cada vez más, el sistema educativo al mundo «de la vida y del desarrollo». De esta forma, se necesitaba una «revisión total del sistema escolar» en el que «la Universidad fuese la orientadora de todo el sistema educativo». En este sentido, la universidad debía fomentar la investigación educativa para que esta redundase sobre la enseñanza primaria y media con el objetivo de que se alejase de un espacio «aristocrático de asilamiento» y se comprometiese con la «sociedad entera $»^{58}$. Es decir, que se debía investigar sobre nuevas técnicas curriculares para unir educación y empleo de una forma que no se había hecho hasta la fecha. Así, se iniciaron investigaciones educativas más acabadas desde el punto de vista teórico ${ }^{59}$. En cierta medida, dichos trabajos venían a recoger el testigo de algunas investigaciones que se habían realizado en el Centre d'études et de recherches pour la diffusion du français francés y que también fueron publicadas en España a modo de ejemplo a seguir ${ }^{60}$.

Por otro lado, el CEDODEP no paró de fomentar esta innovación curricular durante la década. En 1962 dicha institución reconstruía su propia estructura organizativa y creaba un nuevo departamento denominado «Material Didáctico». Entre sus cometidos estaba «estudiar modelos nacionales y extranjeros de material didáctico de toda clase $»^{61}$. En el mismo, se daba «especial» relevancia a todo aquello que estuviese relacionado con los «medios audiovisuales». De esta forma, en este mismo año publicaba un monográfico en su revista, Notas y Documentos, en el que se reproducía el «Informe de reunión de expertos» de la UNESCO celebrada en París sobre «Nuevos métodos y técnicas de educación $»^{62}$. En los años sucesivos, los seminarios y publicaciones en torno a la TV educativa fueron en aumento y aparecieron títulos como Las técnicas

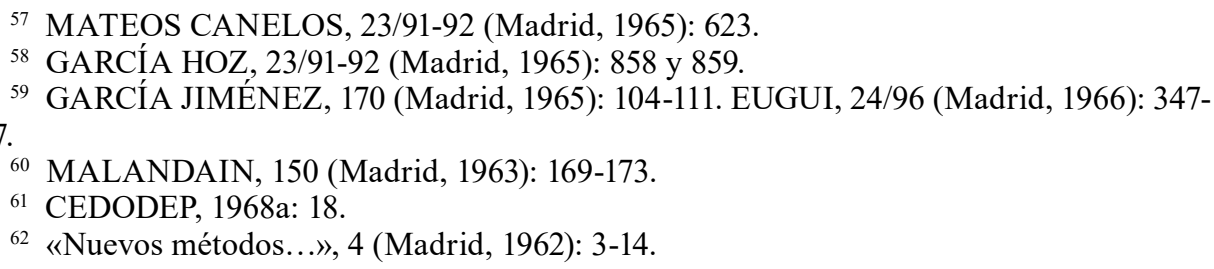


audiovisuales, Los medios audiovisuales en la escuela, Ideas en orden a la introducción en la escuela de las técnicas audiovisuales, Tecnología audiovisual y educación, El Circuito Cerrado de televisión y la formación del profesorado y, en colaboración con Televisión Española, La Televisión Escolar en España, 1967-196863. También debemos sumar otro tipo de iniciativas como la edición del Boletín de Medios Audiovisuales (MAVES), para intentar promover entre el profesorado lo que se entendía como un «eficacísimo apoyo de la labor magistral $»^{64}$.

Sin embargo, este mayor énfasis en el uso de la TV educativa como elemento de desarrollo económico, también estaba relacionado con el vínculo más estrecho que la UNESCO había establecido entre ambos aspectos durante la segunda mitad de los años sesenta. Como ha señalado Emile McAnany, desde finales de los años cuarenta la UNESCO se había convertido en un "rápido consumidor $\rangle^{65}$ de la literatura estadounidense sobre medios de comunicación y desarrollo. Con la publicación de los primeros trabajos del profesor de la Stanford University Wilbur Schramm, rápidamente la UNESCO giró hacia el enfoque de la modernización económica a través del uso de la TV en el sistema educativo. Así, en el año 1964 la UNESCO decidió publicar, junto con la editorial de la Stanford University, el libro de Schramm donde delimitaba la clara relación que podía existir entre mass media, educación y desarrollo económico $^{66}$. Ello dejaba entrever, como ha indicado McAnany, que la UNESCO y otros organismos internacionales, como la Fundación Ford, tuvieron «una fuerte dirección e interés» por lanzar proyectos de TV como modelo curricular sobre el que debía pivotar todo el sistema educativo ${ }^{67}$. Así, durante esta década se llevaron a cabo diferentes proyectos de TV educativa en países como Samoa Americana, El Salvador, Tailandia o Perú, bajo la supervisión de Schramm ${ }^{68}$.

De esta manera, España no iba a ser menos y también se apuntó al desarrollo del primer y único modelo de televisión educativa con programación propia dentro de las aulas que se ha producido en el país ibérico. El programa, denominado TV Escolar, se creó en 1968. Se trataba de una iniciativa llevada a cabo entre el MEN, Televisión Española y el CEDODEP y que tenía su origen en el Primer Plan de Desarrollo. El objetivo, como ya se ha indicado, era que se produjese una «progresiva introducción de las ayudas audiovisuales en el ámbito docente» ${ }^{69}$, para conseguir un aprendizaje más eficaz del alumnado que

${ }^{63}$ CEDOCEP, 1964; 1968b; 1969. NAVARRO HIGUERAS, 1967; 1970. COMISIÓN DE RADIOTELEVISIÓN EDUCATIVA, 1968.

64 «Propósitos», 1 (Madrid, 1966): 2.

${ }_{65}$ MCANANY, 2012: 20.

${ }^{66}$ SCHRAMM, 1964.

${ }^{67}$ MCANANY, 2012: 25.

68 SCHRAMM, COOMBS, KAHNERT y LYLE, $1967 \mathrm{a}$.

${ }^{69}$ TENA ARTIGAS, 95 (Madrid, 1968): 18. 
redundase en una mejora del capital humano y del desarrollo económico. Para conseguirlo, el MEN creó un programa dentro de TVE que debía emitirse en horario escolar (entre las 11:00 y las 12:00 am) para que las escuelas conectasen con el teleprofesor durante una hora diaria y recibieran sus enseñanzas entre tres y seis días a la semana. Para ello, el CEDODEP se encargaría de publicar el «Calendario de Programación de TV Escolar», así como los «Guiones didácticos» que debían seguir el profesorado y el alumnado de cada centro ${ }^{70}$. No obstante, TV Escolar no era un programa de TV educativa al uso en los países en vías de desarrollo. Era distinto a las experiencias que se llevaban a cabo de forma paralela en Colombia, El Salvador o Níger. TV Escolar no albergaba un patrón de organización del sistema educativo en el que la TV fuese su elemento curricular estructural. En realidad, tal y como señaló su director, TV Escolar seguía más bien el modelo de los países desarrollados que eran miembros de la Unión Europea de Radiodifusión (UER) y que «habían acreditado su eficacia» en las aulas ${ }^{71}$.

El porqué de esta situación quizá se encuentra en las propias manifestaciones que el director general de Enseñanza Primaria realizó en la apertura de $T V$ Escolar. Joaquín Tena Artigas escribía dicha presentación con mucha cautela y con cierto recelo respecto a las acciones que podía tomar el profesorado. En varias ocasiones a lo largo del escrito señalaba que «el empleo de los auxilios audiovisuales» no debía establecerse como elemento central de la enseñanza. Para Tena Artigas, TV Escolar no ponía en duda «la indiscutible personalidad de la figura del maestro» en las aulas. Desde el MEN, su director general de Enseñanza Primaria sabía bien que otros modelos de TV educativa habían generado «situaciones de recelo y rechazo por parte de los administradores escolares y del profesorado» ${ }^{72}$ en otros países. Sin ir más lejos, la implantación de la TV educativa en El Salvador había producido durante 1968 graves altercados, que originarían el germen de la guerra civil que derrocó la dictadura militar del país centroamericano ${ }^{73}$. Por otro lado, la situación del magisterio español durante esos años tampoco era la mejor. Desde 1967 se habían producido algunas huelgas docentes por temas salariales ${ }^{74}$. De esta forma, TV Escolar fue un proyecto educativo sujeto a un doble proceso de «refracción»" $\rangle^{75}$. Es decir, al mismo tiempo que estaba condicionado por los actores locales, también su origen era fruto del contexto internacional y de las dificultades

\footnotetext{
70 «Calendario...», 95 (Madrid, 1968): 16-18.

${ }^{71}$ GARCÍA JIMÉNEZ, 196 (Madrid, 1968): 88.

72 TENA ARTIGAS, 95 (Madrid, 1968): 18.

73 LINDO-FUENTES y CHING, 2012: 145-182.

${ }^{74}$ GROVES, 2014: 20-53.

75 GOODSON, 25/6 (Londres, 2010): 767-775.
} 
educativas y políticas que los programas de la UNESCO encontraban a la hora de llevarse a la práctica en los países empobrecidos.

Así, TV Escolar apenas duro un par de años. Con la llegada de la Ley General de Educación de 1970 (LGE) ${ }^{76}$, el sistema educativo bajo la dictadura se reestructuró por completo y TV Escolar dejó de emitirse en horario académico. Quizá ello tuvo que ver con lo señalado anteriormente. No obstante, el abandono de TV Escolar también tenía su razón de ser en las propias delimitaciones que la UNESCO había establecido con la perspectiva del planeamiento de la educación. Un enfoque más centrado en la investigación y que veía en la TV educativa una fórmula más apta para la formación del profesorado o una herramienta complementaria que como enfoque que debía organizar todo el sistema escolar.

\section{LA TRANSFORMACIÓN DE LA TV EDUCATIVA: EVOLUCIÓN Y NUEVAS DIREC- CIONES}

A pesar de la rápida desaparición de TV Escolar de las aulas españolas, la TV educativa no se evaporó como herramienta curricular. En torno a los debates sobre la elaboración de la LGE, la TV educativa sufriría un vuelco importante. Ahora, se establecía como técnica de investigación, formación del profesorado $\mathrm{y}$, en algunos casos, como práctica a desarrollar en las aulas a través de un nuevo formato: el Circuito Cerrado de Televisión (CCTV) ${ }^{77}$. Sin embargo, el CCTV no se originaba como instrumento alejado del espacio en el que habían nacido otras propuestas de TV educativa. En realidad, el CCTV venía a ser una fórmula más dentro del marco que veía a la educación como palanca propulsora del desarrollo económico. No obstante, ahora el enfoque era diferente. El CCTV se insertaba de lleno en una línea de intervención que la UNESCO había abierto desde finales de los años cincuenta. Nos referimos al planeamiento de la educación ${ }^{78}$. Para asegurar que los sistemas educativos cumpliesen su cometido para el desarrollo, era necesario establecer investigaciones previas sobre la eficacia de las técnicas educativas que tendrían que utilizarse. Había, por tanto, que «planear» o «planificar» la educación desde la investigación científica. De hecho, los citados trabajos de Schramm se insertaban dentro de este modelo de intervención ${ }^{79}$.

\footnotetext{
${ }^{76}$ Ley 14/1970, de 4 de agosto, General de Educación y Financiamiento de la Reforma Educativa (BOE de 6 de agosto de 1970).

${ }^{77}$ GARCÍA JIMÉNEZ, 182 (Madrid, 1966): 88-92. NAVARRO VICEDO, 171-172 (Madrid, 1970): 167-181. MIALARET, 17 (Madrid, 1966): 25-28. $2-44$.

78 BOUSQUET, 201 (Madrid, 1968): 43-47. PAREDES GROSSO, 27 (Madrid, 1970):

79 SCHRAMM, COOMBS, KAHNERT y LYLE, $1967 \mathrm{~b}$.
} 
Entre los instrumentos que podían servir para mejorar la educación a través de la investigación estaba el CCTV.

El problema radicaba en que España nunca había establecido una reforma educativa bajo el principio del planeamiento de la educación. Las diferentes reformas que se habían producido bajo el franquismo solían aparecer de manera un tanto desconectada del ámbito de la investigación universitaria ${ }^{80}$. Sin embargo, con los debates previos a la puesta en marcha de la LGE, este principio se intentó resolver. De hecho, la LGE estuvo en todo momento supervisada por el «Comité Asesor Internacional para la Reforma» de la UNESCO en el que se encontraba Philip Coombs ${ }^{81}$. En una reunión secreta, que se produjo en Madrid y Toledo entre el 3 y el 7 de marzo de 1969, el Comité Asesor emitió un informe de recomendaciones. Entre las directrices indicadas, se señalaba la necesidad de dar «prioridad a la investigación educativa» para producir «una transformación de un sistema tradicional» que estaba centrado en un modelo de «rígidas estructuras sociales injustas y opuestas al desarrollo». Para ello, había que utilizar, entre otros aspectos, la «moderna tecnología» ${ }^{82}$.

Por otro lado, la visión de utilizar la TV educativa como una herramienta de investigación para el planeamiento de la educación no sólo fue un enfoque promovido por la UNESCO. En los años finales de la década de los sesenta, el campo educativo español había cambiado. En él, se estableció un debate sobre la necesidad de transformar los modelos de Pedagogía clásica hacia las Ciencias de la Educación ${ }^{83}$. Es decir, acercarse a las líneas de trabajo que se hacían en Francia y que eran más cercanas a la investigación y al planeamiento educativo $^{84}$. Unas direcciones en las que el método curricular del CCTV era muy popular y se utilizaba de manera amplia. Así, en torno a las discusiones sobre la LGE se decidió que el modelo a seguir debía ser ese ${ }^{85}$. Se trataba de construir un espacio de investigación en la universidad que «planease» y formase al profesorado de enseñanzas primaria y secundaria para poder acercar el sistema escolar a los objetivos de desarrollo económico. La receta que se ideó fue denominada red CENIDE-Institutos de Ciencias de la Educación (CENIDE-ICEs).

Como ya hemos señalado en otros trabajos ${ }^{86}$, en realidad el CENIDE fue una institución promovida por diferentes organismos internacionales como

${ }^{80}$ MAINER, 2009.

${ }^{81}$ Carta de Ricardo Díez Hochleitner a D. Gabriel Betancur, Presidente del Comité Asesor Internacional para la reforma de la Educación en España, 17 de septiembre de 1969, ACNEC-UNESCO, caja 342/carpetilla 2.

${ }^{82}$ Informe de la reunión del día 3 de marzo de 1969. Comité de Cooperación Internacional para la reforma Educativa en España, ACNEC-UNESCO, caja 342/carpetilla 2: 2 y 3.

83 «¿Pedagogía...», 186 (Madrid, 1967): 28-29.

84 JOVER OLMEDA y THOILLIEZ RUANO, 2011.

${ }^{85}$ BOUSQUET, 209 (Madrid, 1970): 15-18.

${ }^{86}$ GONZÁLEZ-DELGADO y GROVES, 4/2 (Salamanca, 2017): 73-100. 
la UNESCO y el Programa de las Nacionales Unidas para el Desarrollo (PNUD). Denominado «SPANED 19», era un proyecto de asistencia técnica requerido por la dictadura para el desarrollo económico ${ }^{87}$. La creación del CENIDE en 1969, por tanto, se encuadró dentro de un plan más amplio de desarrollo, junto con otros proyectos concedidos por el PNUD como «Postal services», "Air pollution in Bilbao district» o «Scientific study of water resources in the Canary Island». Incluso, se solicitó un proyecto específico sobre «Educational Televisión», que se fusionaría con los trabajos de investigación del CENIDE. Para conseguir ese objetivo, el CENIDE se constituyó como ente federativo que se encargaba de coordinar los ICEs que se habían creado en las diferentes universidades españolas en torno a la «triple responsabilidad de investigación, formación y experimentación ${ }^{88}{ }^{8}$. En torno a estos objetivos, se comenzaron a desarrollar toda una serie de seminarios de formación del profesorado, proyectos de investigación y compra de material técnico. Dentro de esta rama de actividades, las investigaciones para el planeamiento de la educación a través del CCTV fueron ampliamente promovidas ${ }^{89}$.

Sin embargo, para poder llegar a construir un sistema educativo en conexión con el desarrollo económico no sólo se impartieron seminarios de investigación. El uso del CCTV necesitaba de una amplia formación por parte del profesorado, así como de la compra de equipos para llevarlo a cabo. Por este motivo, a través de la UNESCO, el CENIDE comenzó a poner en marcha un amplio programa de becas para la realización de estancias en el extranjero ${ }^{90}$. Por otro lado, en 1969 esta misma institución, a través de su ATP UNESCO (Bousquet), se puso en contacto por carta con el director del Centre d'Electronique de Massey, de Francia. El propósito de dicho contacto era adquirir un equipo de CCTV para el CENIDE ${ }^{91}$. Con unos fondos aproximados de 1.200.000 pesetas cedidos por el BM, el Ministerio de Educación y Ciencia (MEC) inició la puesta en marcha del proyecto. Pocos meses después, Bousquet se reunió con la editorial española Vicens Vives para que elaborase un informe sobre las posibilidades de enseñanza del Sistema EVR de TV educativa. En ese informe de carácter confidencial, la citada editorial señalaba que el modelo descrito «nos permitiría poner en manos de los países Iberoamericanos un avance tan

${ }^{87}$ Country and Intercountry Programming. UNDP assistance requested by the Goverment of Spain period 1972-1976, ACNEC-UNESCO, caja 75/carpetilla 1: 15.

${ }_{88}$ Decreto de creación del CENIDE de 1969, ACNEC-UNESCO, caja 75/carpetilla 1: 35.

${ }^{89}$ CENIDE, 209 (Madrid, 1970): 61-65.

${ }^{90}$ Comisión Nacional Española de Cooperación con la UNESCO. Secretaría General. Estudio sobre el programa de becas UNESCO-España 1953-1975, Madrid, Junio de 1976, ACNEC-UNESCO, caja 75/carpetilla 1: 3.

${ }_{91}$ Carta de J. Comiran a Monsieur Bousquet Secretaría General Técnica, 18 de diciembre de 1969, ACSEE, caja 61765: Documentación sobre el CENIDE año: 1969-1976.

Hispania, 2020, vol. LXXX, n. ${ }^{\circ} 265$, mayo-agosto, págs. 597-627, ISSN: 0018-2141, e-ISSN: 1988-8368 https://doi.org/10.3989/hispania.2020.017 
importante en los medios de comunicación y la enseñanza, que no dudamos en calificar como uno de los adelantos que revolucionarán el decenio de los $70 »^{92}$. El resultado fue la recomendación de Bousquet al Secretarío General Técnico del MEC para que se apresurara a «encargar e instalar cuanto antes los circuitos cerrados de TV correspondientes $\rangle^{93}$.

Sin embargo, la compra e importación de equipos tecnológicos no se producía de forma aislada. La puesta en marcha del CCTV y otras Tecnologías de la Información y Comunicación (TIC) necesitaban de la presencia de investigadores versados en la materia. Esta era la principal fórmula utilizada para acelerar las actividades de investigación dentro de dicho centro y conseguir los objetivos de modernización educativa. Así, el CENIDE también solicitó a la UNESCO y al PNUD el intercambio/invitación de expertos internacionales. En un programa designado «SPA 19.04.93» se aprobó la llegada de expertos de diferentes disciplinas entre los años 1969 y 1972. La mayoría de los consultores que se demandaban estaban relacionados con temas relativos a la innovación educativa. Un aspecto que, por esos años, era sinónimo de CCTV, enseñanza programada y, sobre todo, «Computer Aided Instruction» $(\mathrm{CAI})^{94}$.

A partir de aquí, comenzaron a aparecer las primeras investigaciones españolas sobre el uso del CCTV como modelo curricular. En todas ellas, se destacó que «su eficacia está ya fuera del terreno de lo hipotético»» ${ }^{95}$. El CCTV podía funcionar perfectamente como un método en el que el teleprofesor evitaba «los estilos de los distintos maestros», así como «los avances y retrasos en el ritmo del cumplimiento del programa» o las «taras propias del individualismo imperante del sistema tradicional $»^{96}$. Incluso, se destacaron las bondades del modelo respecto a que «se puede estudiar con detalle las reacciones más significativas de los alumnos», la «reducción del tiempo en las lecciones impartidas» y que el profesor podía «ver su propia lección y discutirla» para estudiar sus fallos a través del «rebobinado $\rangle^{97}$. De esta manera, las investigaciones se multiplicaros durante los primeros años setenta ${ }^{98}$. Incluso, el CENIDE encargó al ICE de la Universidad de Valencia el desarrollo de una investigación cuyos

${ }_{92}$ Informe sobre el Sistema EVR y sus posibilidades para la enseñanza. Estrictamente confidencial, ACSEE, caja 61765: Documentación sobre el CENIDE año: 1969-1976.

${ }_{93}$ Carta de J. Bousquet ATP CENIDE vía J. Manuel Paredes Secretario General CENIDE a Ilmo. Sr. Pedro Segú y Martín Secretario General Técnico del MEC, 30 de marzo de 1970, ACSEE, caja 61765: Documentación sobre el CENIDE año: 1969-1976.

${ }^{94}$ SPA.019.4.93. Plan de Operaciones España. Centro Nacional de Investigaciones para el desarrollo de la Educación, AGA, caja 61709: Proyectos y programas de la UNESCO, 19691979.

95 SMITH VERDIER, 31/121 (Madrid, 1973): 101.

96 NAVARRO VICEDO, 171-172 (Madrid, 1970): 174.

97 RUIZ HEREDERO, 182-183 (Madrid, 1971): 390.

98 «Microenseñanza», 219 (Madrid, 1972). 
informes tuvieron «un total de cinco volúmenes con 1.500 páginas y cuatro cintas magnetoscópicas $\rangle^{99}$.

Es cierto que los modelos de CCTV fueron importantes durante estos años. No obstante, este tipo de instrumentos para el desarrollo del currículum pronto desaparecieron. Como hemos señalado anteriormente, el CAI se posicionó de manera paulatina como la principal técnica para mejorar el currículum de cara al planeamiento educativo. Existen distintos aspectos que pueden explicar dicha variación. Durante el año 1972, el CENIDE estuvo sujeto a una inspección por parte de especialistas de la UNESCO para observar el funcionamiento de dicho centro. En el informe que se emitió, la misión recomendó encarecidamente dos cosas. La primera, tenía que ver con su evolución institucional. La inspección se mostraba preocupada por la centralización del CENIDE y su actuación como un «súper-ICE en el plano nacional»" ${ }^{100}$. Sería un aspecto que transformó el CENIDE en el Instituto Nacional de Ciencias de la Educación (INCIE) $^{101}$. La segunda, se refería a la necesidad de desarrollar el planeamiento educativo bajo las técnicas más modernas de enseñanza. Los expertos recomendaron que el centro prestase «particular atención a la ejecución del proyecto CAI como una tarea de alcance nacional $»^{102}$. De esta forma, el CAI se observaba como un modelo curricular más adecuado para fomentar las habilidades académicas y productivas del alumnado.

De hecho, el propio CENIDE, ya en su evolución posterior como INCIE, solicitó de nuevo la asistencia de consultores de CAI para impartir nuevos cursos de formación para el profesorado. Durante 1973, dentro del proyecto designado como «UNDP/SPA/19», comenzaron a llegar especialistas de CAI como C. H. Cook y J. R. Hardley, de la Universidad de Leeds; A. Bernstein, de la State University of New York; o H. E. Mitzel, de la Pensilvania State University ${ }^{103}$. Además, dentro del mismo proyecto, también se solicitaba la compra de un «equipo de computación para el proyecto de Enseñanza Asistida por Ordenador (CAI) $\rangle^{104}$. Por otro lado, las recomendaciones sobre el

99 INCIE, 1975: 13.

100 Asistencia al Centro Nacional de Investigaciones para el Desarrollo de la Educación, Madrid. (Proyecto SPANED-19). Informe de la Misión de Inspección, septiembre de 1972, ACNEC-UNESCO, caja 75/carpetilla 1: 19.

101 GONZÁLEZ-DELGADO y GROVES, 4/2 (Salamanca, 2017): 89.

102 Asistencia al Centro Nacional de Investigaciones para el Desarrollo de la Educación, Madrid. (Proyecto SPANED-19). Informe de la Misión de Inspección, septiembre de 1972, ACNEC-UNESCO, caja 75/carpetilla 1: 23.

${ }^{103}$ Consultores UNESCO. Proyecto UNDP/SPA/19 CENIDE, 18 de enero de 1973, ACSEE, caja 61709: Proyectos y Programas de la UNESCO.

${ }^{104}$ Carta del Jefe de la Sección del CENIDE a D. Manuel Utande Igualada Director de la Unidad Administrativa del Programa del Banco Mundial, 31 de octubre de 1973, ACSEE, caja 61709: Proyectos y Programas de la UNESCO.

Hispania, 2020, vol. LXXX, n. ${ }^{\circ}$ 265, mayo-agosto, págs. 597-627, ISSN: 0018-2141, e-ISSN: 1988-8368 https://doi.org/10.3989/hispania.2020.017 
giro hacia estas nuevas direcciones también llegaban por el lado oficial de la UNESCO. Durante estos años, el citado organismo publicó una serie de informes sobre la necesidad de doblar los esfuerzos en utilizar el ordenador como modelo curricular ${ }^{105}$.

Sin embargo, la paulatina desaparición de la TV como ámbito curricular sobre el que asentar el desarrollo económico no puede explicarse exclusivamente por las variaciones internas de los informes y posiciones de los expertos internacionales. La década de los setenta fue una época convulsa desde diferentes puntos de vista para el sistema educativo español. Justo en el momento en el que la TV educativa parecía estar en su momento más álgido, se produjeron varios acontecimientos que hicieron variar su sentido dentro del campo educativo español. El primero de ellos, tuvo que ver con el crecimiento de los movimientos de enseñantes y los grupos educativos alternativos en los últimos años de la dictadura. Tales grupos, como los Movimientos de Renovación Pedagógica (MRPs), establecieron una crítica constante hacia los modelos educativos de la LGE y plantearon espacios de construcción curricular alternati$\operatorname{vos}^{106}$. Estos debates facilitaron, entre otras cosas, la construcción de una amplia crítica a las pedagogías de carácter técnico ${ }^{107}$. Segundo, la decadencia del régimen también abrió el frente de discusión dentro del campo académico de las ciencias de la educación. Diferentes áreas como la historia de la educación, la psicología de la educación o la sociología de la educación emprendieron proyectos propios. Tal división abrió nuevas líneas de investigación y limitó la posibilidad de dirigir el campo educativo desde una posición unívoca ${ }^{108}$. Tercero, y quizá lo más importante, durante los años setenta se estableció una crítica abierta a nivel mundial sobre las teorías de la modernización ${ }^{109}$. Por primera vez se empezaron a desarrollar importantes estudios que ponían en duda la relación entre sistema educativo, TV y crecimiento económico ${ }^{110}$. No debemos perder de vista que, en efecto, la teoría de la modernización llevaba implícita una concepción del desarrollo muy apegada a los principios políticos de corte libera ${ }^{111}$. Una perspectiva crítica que también tuvo su impulso en España a través de las teorías de la reproducción y un cambio de demandas por parte de los actores sociales hacia el sistema educativo ${ }^{112}$.

105 UNESCO, 1974.

106 GROVES, 2014.

107 GIMENO SACRISTÁN, 1982.

108 VIÑAO, 2005.

109 GILMAN, 2003.

110 ARNOVE, 25/2 (Washington, 1975): 144-156. CARNOY, 40/240 (Nueva York, 1975): 370-384.

111 Más información sobre la visión política desde la que partía la teoría de la modernización en FERGUSON, 1990.

112 LERENA ALESÓN, 1976. 


\section{CONCLUSIONES}

La TV educativa fue un modelo curricular que estuvo muy presente en el espacio educativo franquista. Sin embargo, su origen no hay que buscarlo en los intentos del régimen por establecer un fenómeno de adoctrinamiento de los valores del nacionalcatolicismo. Tampoco debe entenderse como una tentativa curricular que se implantaba para legitimar y «lavar la cara» del país ibérico en la esfera internacional. Su inicio se encuentra en el espacio abierto por la UNESCO para intentar conseguir, a través de la educación, que los países en vías de desarrollo modernizaran su estructura económica. La dictadura se había sumado al concepto de la educación que el bloque occidental había abierto en el contexto de la Guerra Fría. Esta es la causa que explica por qué durante el franquismo se desarrollaron este tipo de políticas.

Desde los inicios de la década de los cincuenta, cuando se fomentaron estas iniciativas en el ámbito internacional, el campo educativo español comenzó a desarrollar propuestas en esta dirección. En un primer momento, se centraron los esfuerzos en erradicar el analfabetismo. Para ello, vieron en la TV educativa una posible técnica que podía ayudar a solucionar los problemas de las zonas rurales, al tiempo que se presentaba como una política curricular que favorecía la modernización del sistema escolar. De esta forma, la Comisión de Extensión Cultural y la Junta Nacional contra el Analfabetismo desplegaron sus iniciativas en el marco del Programme of Fundamental Education de la UNESCO. Sin embargo, las iniciativas no se quedaron en este punto. Tanto el MEN como el Ministerio de Información y Turismo lanzaron proyectos de modernización a través de la TV educativa para intentar renovar el sistema educativo español y sus espacios culturales y económicos. Los Teleclubs, las iniciativas del CEDODEP y de TVE fueron buen ejemplo de ello.

A partir de aquí, este tipo de propuestas se acelerarían. Las teorías de la modernización ampliaban su margen discursivo y, con ello, también sus fundamentos prácticos. En los inicios de la década de los sesenta, los organismos internacionales proponían al régimen que doblara sus esfuerzos por ampliar el marco de escolarización de todos los niveles de enseñanza, como una forma de alcanzar el desarrollo económico. Para ello, la TV educativa podía funcionar como una política curricular fundamental. Así, el régimen convergió con este tipo de política y pronto creó el Centro Nacional de Enseñanza Media por Radio y Televisión, al tiempo que la investigación sobre el uso de la TV en la escuela se multiplicaba. Se podría decir que el discurso de la modernización construyó una especie de imaginario dentro de la dictadura que absorbió y superó las posibles reticencias que algunos grupos pudieron mostrar a este tipo de políticas. Ello no quiere decir que los valores del nacionalcatolicismo desapareciesen. Ahora bien, la concepción del sistema educativo como pilar básico para alcanzar los fines del desarrollo era la misma que expresaban los 
organismos internacionales. Un aspecto que dejaba en un segundo plano los valores tradicionales de la dictadura.

Esta dirección fue a más y, a finales de la década de los sesenta, España había creado un programa propio de TV educativa (TV Escolar). Sin embargo, su duración fue escasa. Los motivos de su desaparición fueron varios. Quizá, el principal hace referencia a la asimilación del enfoque del planeamiento de la educación propuesto, de nuevo, por la UNESCO. Así, bajo el seno del CENIDE y el INCIE, el campo educativo español inició toda una serie de programas, seminarios y experiencias de investigación a través del CCTV. En realidad, y al igual que los programas previos de TV educativa, el CCTV también tenía como fin último conseguir que el sistema educativo estuviese más acorde con los objetivos del desarrollo económico. Su rápida desaparición, también nos habla de las crisis del modelo en dos vertientes. La primera, relacionada con el nacimiento de otras técnicas curriculares como las computadoras. La segunda, una ruptura del régimen y una crisis a nivel mundial de las teorías de la modernización. Sea como fuere, lo importante a destacar en este punto es que la TV educativa durante la dictadura, en tanto que modelo curricular, debe insertarse en los debates abiertos por los organismos internacionales y una concepción concreta sobre el sistema educativo. Su origen, así como sus direcciones y evoluciones posteriores, sólo es entendible si lo situamos bajo este marco o perspectiva de análisis.

\section{Bibliografía}

Agulló Díaz, M. ${ }^{a}$ Carmen y Payà Rico, Andrés, «Democracia, renovación pedagógica y territorio rural: la escuela agrícola comarcal valenciana "La Serranía"», Historia y Memoria de la Educación, 7 (Madrid, 2018): 299-334.

Álvaro-Moya, Adoración, «Guerra Fría y formación de capital humano durante el franquismo. Un balance sobre el programa estadounidense de ayuda técnica (19531963)», Historia del Presente, 17 (Madrid, 2011): 13-25.

Antona, Tamara, «Los orígenes de la Televisión Educativa en TVE (1958-1966)», Estudios sobre el Mensaje Periodístico, 20 (Madrid, 2014): 209-226.

Arnove, Robert, «Sociopolitical Implications of Educational Television», Journal of Communication, 25/2 (Washington, 1975): 144-156.

Boel, Jens, «UNESCO’s Fundamental Education Program, 1946-1958: Vision, Actions and Impact», en Paul Duedahl (ed.), The History of UNESCO: Global Actions and Impacts, Londres, Palgrave-Macmillan, 2016: 153-167.

Borreguero-Sierra, M. ${ }^{\text {a }}$ Concepción, «La televisión en las zonas rurales del Japón», Revista de Educación, 147 (Madrid, 1962): 31-34.

Bousquet, Jacques, «El precio de la educación», Revista de Educación, 88 (Madrid, 1958): 25-29.

Hispania, 2020, vol. LXXX, n. ${ }^{\circ}$ 265, mayo-agosto, págs. 597-627, ISSN: 0018-2141, e-ISSN: 1988-8368 https://doi.org/10.3989/hispania.2020.017 
Bousquet, Jacques, «Televisión y Educación (I)», Revista de Educación, 73 (Madrid, 1958): 35-38.

Bousquet, Jacques, «Televisión y Educación (II)», Revista de Educación, 74 (Madrid, 1958): 64-68.

Bousquet, Jacques, «Tendencias del planeamiento educativo en 1968», Revista de Educación, 201 (Madrid, 1968): 43-47.

Bousquet, Jacques, «Las puertas del futuro», Revista de Educación, 209 (Madrid, 1970): 15-18.

«Calendario de Programación de TV Escolar», Vida Escolar, 95 (Madrid, 1968): 16-18.

Carnoy, Martin, «The economic costs and returns to educational television», Ekistics, 40/240 (Nueva York, 1975): 370-384.

Cassirer, Henry, «Trying out Televisión in Japanese Classrooms», UNESCO Courier 6/12 (París, 1953): 17-18.

Cebrián Villar, Mar y López, Santiago M., «La dimensión internacional de las transformaciones en la política científica y el cambio técnico» en Lorenzo Delgado Gómez-Escalonilla, Ricardo Martín de la Guardia y Rosa Pardo Sanz (eds.), La apertura internacional de España. Entre el franquismo y la democracia (19531986), Madrid, Silex, 2016: 233-261.

CEDODEP, Las técnicas audiovisuales. Documento cicloestilado, 17, Madrid, CEDODEP, 1964.

CEDODEP, El Centro de Documentación y Orientación Didáctica: Diez años de actividades (1958-1968), Madrid, MEN-CEDODEP, 1968a.

CEDODEP, Ideas en orden a la introducción en la escuela de las técnicas audiovisuales, Madrid, CEDODEP, $1968 \mathrm{~b}$.

CEDODEP, Tecnología audiovisual y educación, Madrid, CEDODEP, 1969.

CENIDE, «Actividades del CENIDE», Revista de Educación, 209 (Madrid, 1970): 61-65.

Comisión de Radiotelevisión educativa, La Televisión Escolar en España, 1967-1968, Madrid, RTVE-Dirección de Enseñanza Primaria, 1968.

Corrales Morales, David, «Much Ado about Nothing? Lights and Shadows of the World Bank's Support of Spanish Aspirations to Educational Modernization (19681972)», en Óscar J. Martín García y Lorenzo Delgado Gómez-Escalonilla (eds.), Teaching Modernization Spanish and Latin American Educational Reform in the Cold War, New York, Berghahn Books, 2020: 127-149.

Costa Riba, José, «Problemas pedagógicos de la Radio y Televisión», Bordón, 78-79 (Madrid, 1958): 369-384.

De Reyna, Guillermo, «Los métodos audiovisuales en la Educación Fundamental. Notas al congreso de Milán», Revista de Educación, 1/2 (Madrid, 1952): 164-167.

De Sobrino, José, «Una universidad por televisión en los Estados Unidos», Revista de Educación, 4/9 (Madrid, 1953): 47-49.

Del Pozo Andrés, M. ${ }^{a}$ del Mar, "Community and the myth of the ideal school: circulation and appropriation of the Hamburg Gemeinschaftsschulen in Spain (19221933)», Paedagogica Historica, 50/5 (Londres, 2014): 599-614. 
Delgado Gómez-Escalonilla, Lorenzo, «Modernizadores y tecnócratas. Estados Unidos ante la política educativa y científica de la España del Desarrollo», Historia y Política, 34 (Madrid, 2015): 113-146.

Dieuzeide, Henri, «La Televisión Escolar Francesa», Bordón, 78-79 (Madrid, 1958): 405-414.

Dorn, Charles y Ghodsee, Karen, «The Cold War Politicization of Literacy: Communism, UNESCO, and the World Bank», Diplomatic History 36/2 (Oxford, 2012): 373-398.

Dumazadier, Joffre, Television and Rural Adult Education: The Tele-clubs in France, París, UNESCO, 1956.

«Editorial», Bachillerato RTV. Revista de Orientación Didáctica e Información Escolar. Primer Curso, 1 (Madrid, 1963): 1.

Eugui, Julio, «La Televisión escolar ante un gran futuro», Revista Española de Pedagogía, 24/96 (Madrid, 1966): 347-357.

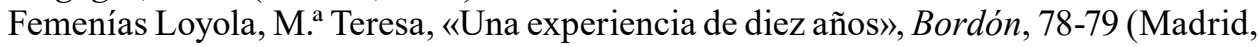
1958): 415-424.

Ferguson, James, The Anti-Politics Machine: 'Development', Depoliticization and Bureaucratic Power in Lesotho, Cambridge, Cambridge University Press, 1990.

García, Luis, «Cuadernos de educación fundamental», Revista Española de Pedagogía, 18/70 (Madrid, 1960): 207-208.

García Hoz, Víctor, «Algunos números sobre la educación en sus relaciones con la vida económica y los medios de comunicación», Bordón, 107-108 (Madrid, 1962): 127-136.

García Hoz, Víctor, «Discurso de clausura», Revista Española de Pedagogía, 23/91-92 (Madrid, 1965): 845-862.

García Jiménez, Jesús, «La enseñanza de la religión por radio y televisión», Revista Española de Pedagogía, 21/82-83 (Madrid, 1963): 55-74.

García Jiménez, Jesús, «Medios Audiovisuales y Cultura de Desarrollo» Bordón, 118119 (Madrid, 1963): 379-406.

García Jiménez, Jesús, «La televisión, promesa y amenaza educativas (I): Televisión infantil y vida de familia», Revista de Educación, 170 (Madrid, 1965): 104-111.

García Jiménez, Jesús, «La formación del personal de televisión», Revista de Educación, 182 (Madrid, 1966): 88-92.

García Jiménez, Jesús, «Televisión escolar. Notas informativas acerca de su naturaleza, planteamiento y ejecución», Revista de Educación, 196 (Madrid, 1968): $88-90$.

García-Fernández, Mónica, «Sexualidad y armonía conyugal en la España franquista. Representaciones de género en manuales sexuales y conyugales publicados entre 1946 y 1968», Ayer, 105/1 (Madrid, 2017): 215-238.

Gilman, Nils, Mandarins of the Future: Modernization Theory in Cold War America, Baltimore, The Johns Hopkins University Press, 2003.

Gimeno Sacristán, José, La pedagogía por objetivos. La obsesión por la eficacia, Madrid, Morata, 1982.

González-Delgado, Mariano y Groves, Tamar, «La enseñanza programada, la UNESCO y los intentos por modificar el curriculum en la España desarrollista (1962-1974)», Espacio, Tiempo y Educación, 4/2 (Salamanca, 2017): 73-100. 
Gonzalvo Mainar, Gonzalo, «El texto escolar y la didáctica moderna», Bordón, 92-93 (Madrid, 1960): 247-54.

Goodson, Ivor, «Times of educational change: Towards an understanding of patterns of historical and cultural refraction», Journal of Education Policy, 25/6 (Londres, 2010): 767-775.

Groves, Tamar, Teachers and the Struggle for Democracy in Spain, 1970-1985, Londres, Palgrave-Macmillan, 2014.

Hernández Díaz, José M. (ed.), Influencias suizas en la educación española e iberoamericana, Salamanca, Ediciones de la Universidad de Salamanca, 2016.

INCIE, Circuito cerrado de televisión y enseñanza, Madrid, MEC, 1975.

Jones, Philip W. y Coleman, David, The United Nations and Education Multilateralism, Development and Globalization, Nueva York, Routledge-Falmer, 2005.

Jover Olmeda, Gonzalo y Thoilliez Ruano, Bianca, "La pedagogía "au pluriel” y su incidencia en la reformulación del conocimiento teórico de la educación en España», en José María Hernández Díaz (ed.), Francia en la educación de la España contemporánea (1808-2008), Salamanca, Universidad de Salamanca, 2011: 219-247.

León-Aguinaga, Pablo, «Faith in the USA. El mensaje de la diplomacia pública americana en España, 1948-1960», en Antonio Niño y José A. Montero (eds.), Guerra Fría y Propaganda. Estados Unidos y su cruzada cultural en Europa y América Latina, Madrid, Biblioteca Nueva, 2012: 197-234.

Lerena Alesón, Carlos, Escuela, Ideología y Clases Sociales en España: Crítica de la sociología empirista de la educación, Barcelona, Ariel, 1976.

Lindo-Fuentes, Héctor y Ching, Erik, Modernizing Minds in El Salvador: Education Reform and the Cold War, 1960-1980, Albuquerque, University of New Mexico Press, 2012.

Lipset, Seymour M., «Viejas y nuevas fronteras de la Sociología Política», Revista de Estudios Políticos, 100 (Madrid, 1958): 49-94.

Lipset, Seymour M., «Cristalizaciones políticas en las sociedades desarrolladas y en vías de desarrollo», Revista de Estudios Políticos, 139 (Madrid, 1965): 5-40.

Lora Tamayo, Manuel, «España y el Programa 1965-66 de la UNESCO», Revista de Educación, 166 (Madrid, 1964): 53-59.

Maíllo, Adolfo, «Los métodos rápidos de enseñanza de la lectura y la escritura en la Junta Nacional contra el Analfabetismo», Bordón, 54 (Madrid, 1955): 371-374.

Maíllo, Adolfo, «Cuestiones actuales de educación y enseñanza», Revista de Educación, 149 (Madrid, 1963): 114-118.

Mainer, Juan, La forja de un campo profesional. Pedagogía y didáctica de las Ciencias Sociales en España (1900-1970), Madrid, CSIC, 2009.

Malandain, Claudio, «La percepción ante la pantalla y las técnicas audiovisuales», Revista de Educación, 150 (Madrid, 1963): 169-173.

Mallas Casas, Juan, «Uso eficiente de los medios audiovisuales», Vida Escolar, 37 (Madrid, 1962): 32-34.

Martín Alonso, Antonio, «Bases pedagógicas para una didáctica audiovisual», Revista Española de Pedagogía, 23/91-92 (Madrid, 1965): 614-615.

Martín-Antón, Javier, «Una revisión acerca de las salas de televisión en España y su incidencia en Asturias», Espacio, Tiempo y Forma, 29 (Madrid, 2017): 353-390. 
Martín-García, Óscar J., «Una utopía secular. La teoría de la modernización y la política exterior estadounidense en la Guerra Fría», Historia y Política, 34 (Madrid, 2015): 27-52.

Martín-García, Óscar J., "'The Most Developed of the Underdeveloped Nations’. US Foreign Policy and Student Unrest in 1960s Spain», The International History Review, 41/3 (Londres, 2019): 539-558.

Mateos Canelos, Luis, «Televisión y enseñanza de las matemáticas», Revista Española de Pedagogía, 23/91-92 (Madrid, 1965): 622-623.

McAnany, Emile, Saving the World: A Brief History of Communication for Development and Social Change, Baltimore, University of Illinois Press, 2012.

MEN, España y la Unesco. Colaboración al Proyecto Principal 'Extensión y Perfeccionamiento de la Educación Primaria en América Latina, Madrid, MEN, 1962.

Mialaret, Gaston, «La televisión en circuito cerrado y los problemas de la enseñanza», Notas y Documentos, 17 (Madrid, 1966): 25-28.

«Microenseñanza», Revista de Educación, 219 (Madrid, 1972).

Navarro Higueras, Juan, «Medios audiovisuales: Problemas generales», en La Escuela Unitaria Completa, Madrid, MEN/CEDODEP, 1960: 220-226.

Navarro Higueras, Juan, «La grabación magnética», Vida Escolar, 62 (Madrid, 1964): 26-28.

Navarro Higueras, Juan, «Aplicaciones didácticas del magnetófono», Vida Escolar, 65 (Madrid, 1965): 19-21.

Navarro Higueras, Juan (ed.), Los medios audiovisuales en la escuela, Madrid, CEDODEP, 1967.

Navarro Higueras, Juan, «El Circuito Cerrado de televisión y la formación del profesorado», Notas y Documentos, 29-30 (Madrid, 1970).

Navarro Vicedo, M. ${ }^{a}$ Dolores, «La enseñanza por televisión en circuito cerrado. La experiencia docente de Marly Le Roi», Bordón, 171-172 (Madrid, 1970): 167-181.

«Nuevos métodos y técnicas de educación», Notas y Documentos, 4 (Madrid, 1962): 3-14.

OECD, «La educación española en el desarrollo económico», Revista de Educación, 147 (Madrid, 1962): 25-30.

OECD, «OECD: Desarrollo de la enseñanza española», Revista de Educación, 175 (Madrid, 1965): 117

Oliveros, Ángel, XXV años de la Comisión Nacional Española de Cooperación con la UNESCO, Madrid, UNESCO-MEC, 1978.

Ossenbach, Gabriela y Groves, Tamar, «Entre la mitificación y la crítica: el cine y los medios audiovisuales en la escuela primaria en España en el tardofranquismo y la transición, 1958-1982», Cahiers de civilisation espagnole con contemporaine, 11 (París, 2013): 2-17.

Ossenbach, Gabriela y Martínez-Boom, Alberto, «Itineraries of the discourses on development and education in Spain and Latin America (circa 1950-1970)», Paedagogica Historica, 47/5 (Londres, 2011): 679-700.

Otero-Urtaza, Eugenio, «The educational Missions under the Second Republic in Spain (1931-1936): a framework for popular education», en Saak Braster, Frank Simon e Ian Grosvenor, (eds.), A History of Popular Education: Educating the People of the World, Londres, Routledge, 2013: 207-220. 
Paredes Grosso, Juan Manuel, «Métodos de Planificación de la Educación», Notas y Documentos, 27 (Madrid, 1970): 2-44.

Payà Ibars, Raquel, «Los usos de la TV en las actividades formativas», Revista Española de Pedagogía, 23/91-92 (Madrid, 1965): 660-666.

«¿Pedagogía o Ciencias de la Educación?», Revista de Educación, 186 (Madrid, 1967): 28-29.

«Primer concurso sobre guiones radiofónicos», Vida Escolar, 68 (Madrid, 1965): 33. «Propósitos», Boletín Medios Audio Visuales, 1 (Madrid, 1966): 1-3.

Ruiz Heredero, Carmen, «¿De qué modo un circuito cerrado de televisión contribuye a formar profesores?», Bordón, 182-183 (Madrid, 1971): 381-398.

Schramm, Wilbur, Mass Media and National Development: The Role of Information in the Developing Countries, Palo Alto/París, Stanford University Press/UNESCO, 1964.

Schramm, Wilbur, Coombs, Philip, Kahnert, Friedrich y Lyle, Jack, New Educational Media in Action: Case Studies for Planners, París, UNESCO, 1967a, 3 vols.

Schramm, Wilbur, Coombs, Philip, Kahnert, Friedrich y Lyle, Jack, The New Media: Memo to Educational Planners, París, UNESCO, $1967 \mathrm{~b}$.

Smith Verdier, Enrique, «Circuito cerrado de TV y formación del profesorado», Revista Española de Pedagogía, 31/121 (Madrid, 1973): 101-110.

Suárez Rodríguez, Luis, «La utilización de los medios audiovisuales en la enseñanza laboral», Revista Española de Pedagogía, 23/91-92 (Madrid, 1965): 760-761.

Taylor, Duncan, «La labor del Servicio de Radioemisiones Escolares de la BBC», Bordón, 78-79 (Madrid, 1958): 395-404.

Tena Artigas, Joaquín, «Un nuevo instrumento al servicio de la enseñanza: la Televisión Escolar», Vida Escolar, 95 (Madrid, 1968): 18-20.

Terrón, Aida, Comelles, Josep y Perdiguero-Gil, Enrique, «Schools and health education in Spain during the dictatorship of General Franco (1939-1975)», History of Education Review, 46/2 (Sidney, 2017): 208-223.

Tornafoch-Yuste, Xavier y Opisso-Atienza, Isavena, «National identity, civic values and school textbooks in Spain: La enciclopedia cíclico pedagógica and el libro de España (1931- 1957)», History of Education \& Children's Literature, 10/1 (Macerata, 2015): 361- 367.

Trölher, Daniel, «The technocratic Momentum after 1945, the Development of Teaching Machines, and Sobering Results», Journal of Educational, Media, Memory, and Society, 5/2 (Londres, 2013): 1-19.

UNESCO, «La Televisión: Oportunidad y problema educativo», El Correo de la UNESCO, 6/3 (París, 1953).

UNESCO, Developing mass media in Asia: papers of a UNESCO meeting at Bangkok, París, UNESCO, 1960.

UNESCO, Film and television in the service of opera and ballet and of museums: reports on two international meetings, París, UNESCO, 1961.

UNESCO, New trends in the utilization of educational technology for science education, París, UNESCO, 1974.

Viñao, Antonio, «La Pedagogía como Ciencia, Arte y Profesión en la España del siglo $\mathrm{XX}$ », en Un siglo de Pedagogía cientifica en la Universidad Complutense de Madrid, Madrid, Universidad Complutense, 2005: 55-62. 
Viñao, Antonio, «Reformas e innovaciones educativas en la España del primer tercio del siglo XX. La JAE como pretexto», Revista de Educación, número extraordinario (Madrid, 2007): 21-44.

Warleta-Fernández, Enrique, «Educación fundamental por radio en Colombia», Bordón, 78-79 (Madrid, 1958): 459-468.

Recibido: 20/02/2019

Aceptado: 29/01/2020 